\title{
Children infect mothers in AIDS outbreak at a Soviet hospital
}

Moscow

Almost the whole Soviet Union has been shocked by the revelation that a single hospital at Elista, the capital city of the Kalmyk autonomous republic (population 344,000 ), has managed to infect no fewer than 27 young children and five of their mothers with the human immunodeficiency virus (HIV).

At the end of last year, there were only about 120 officially registered AIDS cases in the Soviet Union, of whom only six had clinical symptoms of disease.

The immediate cause of the Elista outbreak is the criminal negligence and irresponsible attitude of the medical staff at the children's hospital at Elista, where syringe needles were reused without adequate sterilization.

The outbreak was first recognized late last November, when a new-born baby at the hospital tested positive for HIV. Following established procedures, the case was reported to the Central Institute for Epidemiology in Moscow, whose specialized AIDS laboratory collects information and also examines all suspected cases of HIV infection. Tests on the baby from Elista confirmed suspicions, but both parents tested negative for HIV.

Almost at the same time, the blood donor service at Elista found another virus carrier by HIV screening (routine since the beginning of 1988) - a young woman with a recently dead baby. Although her contacts revealed no further infection, it emerged that her dead child had spent almost a month at the Elista hospital, which prompted an urgent investigation by the Moscow AIDS laboratory.

The outcome stunned the investigators, who examined serum samples from all the children treated at the hospital at the same time as the dead child and the newly infected children. No fewer than 4 out of 19 tests were positive for HIV.

Vadim Pokrovsky, head of the AIDS laboratory, promptly flew to Elista with a team to collaborate with the republic's public health services in screening all children who could have had contact with those infected. One complication is that most child patients at the Elista hospital suffer from chronic diseases and are referred there only when treatment at other general hospitals has been ineffective. But 27 infected children have now been identified.

Knowing that HIV infection can be transferred from mother to new-born child, the investigators expected to find as many infected women as children. But only four of the 27 mothers were found to be infected. So how could the children of uninfected mothers acquire HIV? Most probably, by the use of non-sterile medical instruments and serious breaches of the rules of hygiene at the hospital.

Meanwhile, the medical commission has concluded that the five infected mothers (the four now found and the mother of the dead child) must have been infected by their children. The 'external' contacts of the four women are acknowledged to be clear, and screening of 12,000 Elista residents has turned up no further cases. One possibility is suggested by the observation that many of the children treated at Elista are weak, with stomatitis and bleeding gums, and that HIV may be transmitted from child to mother in breast-feeding via cracks in the nipple.

While it is too soon to draw final conclusions about the Kalmyk AIDS outbreak, some specialists expect that two or three times as many AIDS cases will eventually be found as are now known.

But the Kalmyk situation says much about the drive against AIDS in the Soviet Union. It is plain that the headstart of several years - the time-lag between the appearance of infection in the United States and the Soviet Union - has not been used to mount effective preparations against an epidemic. While traditional stereotypes have been a brake on the spread of infection, the attitudes of the health authorities have also been an obstacle.

With the restructuring of the health services now under way, top Soviet officials are now in close touch with the World Health Organization and are seeking to erect reliable barriers against an AIDS epidemic. A uniform state-wide programme has been adopted, screening is under way, laboratories are being set up and Soviet-made instruments and diagnostic reagents are being developed. But the task is complicated by laggard industry, the sometimes low skill of medical personnel and the often inadequate state of public health information - the word "condom" made its appearence in the press barely six months ago.

Direct responsibility for the AIDS outbreak at Elista will be determined by the courts, but it must be hoped that the legal investigation will be a turning point for Soviet attitudes towards the AIDS problem

Viktor Belitsky Novosti

\section{Unproved AIDS drug is made available for limited use}

\section{Boston}

THE Food and Drug Administration (FDA) has this week granted limited approval for a drug believed to be effective in preventing Pneumocystis carinii pneumonia, the leading cause of death in AIDS patients. The decision makes good the FDA's commitment last year to bring promising drugs more swiftly to patients with AIDS or other life-threatening illnesses.

The drug, an aerosol version of pentamidine, has been shown in a preliminary and as yet unpublished clinical study at San Francisco General Hospital to inhibit the recurrence of Pneumocystis pneumonia. FDA's authorization allows the use of the drug in "secondary prophylaxis" in patients who have already suffered a bout of the pneumonia. The FDA is also authorizing its use in patients who have not yet contracted the pneumonia provided that their T4 blood-cell count is less than 200 per millilitre of blood. This decision is based solely on preliminary data from a federal study showing that patients with low $T$ blood-cell counts face a particularly great risk of contracting the pneumonia.

Jerome Groopman, an AIDS researcher at the New England Deaconess Hospital in Boston, says that FDA's decision on primary prophylaxis is "important and correct" and that it shows the FDA's willingness to act on the basis of clinical data "which makes sense in practice without necessarily conforming to the highly stringent requirements" of final approval.

The procedure followed in approving the aerosol form of pentamidine, known as the treatment investigational new drug (IND) procedure (see Nature 326, 536; 1987), has been used previously to offer drugs to patients before final licensing. Azidothymidine (AZT), the only drug licensed for use against the AIDS virus HIV, was also at first released as a treatment IND. But this is the first time that the manufacturer is likely to take advantage of a provision allowing it to recover the costs of the drug's dissemination during this period. An announcement about the financial and logistical details, including a special telephone number which physicians and hospitals can call to order the drug, is expected shortly by LyphoMed Inc. of Rosemont, Illinois.

The aerosol version of pentamadine has hitherto been available only in research programmes, most of which are centred on San Francisco and New York. But pentamadine in intravenous form, or by subcutaneous injection, is already licensed and marketed widely as a treatment for Pneumocystis pneumonia.

Seth Shulman 